\title{
Monoclonal antibody profiling of cell surface proteins associated with the viral biofilms on HTLV-1 transformed cells
}

\author{
A. TARASEVICH, A. FILATOV, A. PICHUGIN, D. MAZUROV* \\ Institute of Immunology, Kashirskoe shosse 24-2, Moscow, 115478, Russia
}

Received November 24, 2014; revised March 11, 2015; accepted August 3, 2015

\begin{abstract}
Summary. - Human T lymphotropic virus 1 (HTLV-1) is a pathogenic retrovirus that spreads predominantly via cell-to-cell contact. Two models of cell-to-cell virus transmission are proposed: virological synapse (VS) and viral biofilms (VB). Both infectious structures can be involved in transmission and synergistically enhance HTLV-1 spread between cells. Although transmission of virus via VB has been reported, the molecular composition of VB remains poorly understood. In this study we generated new anti-VB monoclonal antibodies (MAbs) and screened them along with a panel of anti-human cluster of differentiation (CD) MAbs to select antigens associated with VB. Among four MAbs generated against VB, two MAbs were identified as anti-CD25 (IL-2RA). We found that antigens CD4, CD150, CD25, CD70, and CD80 were enriched in VB. We also determined that expression of viral protein Tax, a central molecule in HTLV-1 transmission, upregulates intercellular adhesion molecule 1 (ICAM-1), CD95, CD25, CD70, and CD80. Whether these antigens are essential for VB formation and HTLV-1 infection remains unknown and will be determined in further experiments.
\end{abstract}

Keywords: monoclonal antibody; CD antigen; HTLV-1; biofilms

\section{Introduction}

The group of retroviruses that are pathogenic for humans consists of the oncoretrovirus human $\mathrm{T}$ lymphotropic virus 1 (HTLV-1), and the lentivirus human immunodeficiency virus (HIV). HIV causes the onset of a fatal immunodeficiency in all patients, while only $5 \%$ of individuals infected with HTLV-1 develop either adult T cell leukemia or HTLV-1associated myelopathy/tropical spastic paraparesis (HAM/ TSP). It has been shown that cell-to-cell transmission of

*Corresponding author. E-mail: dvmazurov@yandex.ru; phone: +74996128854 .

Abbreviations: $\mathrm{CD}=$ cluster of differentiation; HIV-1 = human immunodeficiency virus 1 ; HTLV-1 = human T cell lymphotropic virus 1; ICAM-1 = intercellular adhesion molecule 1; IL-2$\mathrm{RA}=$ interleukin 2 receptor subunit $\mathrm{A}$; IP = immunoprecipitation; $\operatorname{MAb}(\mathrm{s})=$ monoclonal antibody(ies); MS = mass-spectrometry; $\mathrm{PE}=$ phycoerythrin; $\mathrm{TM} 4 \mathrm{SF}=$ transmembrane 4 superfamily proteins; $\mathrm{VB}=$ viral biofilms; $\mathrm{VS}=$ virological synapse; $\mathrm{WB}=$ Western blot; $\mathrm{Wt}=$ wild type these viruses mediated by so-called virological synapse (VS) is more efficient mechanisms of viral spread than infection with cell-free virus (Chen, 2012; Igakura et al., 2003; Jolly et al., 2004; Pique and Jones, 2012).

Unlike HIV, HTLV-1 viral particles are assembled into special structures and are transiently stored on the surfaces of infected cells. These structures are reminiscent of bacterial biofilms and called HTLV-1 biofilm-like structures (Pais-Correia et al., 2010). Below we will refer to these structures as viral biofilms (VB). Pais-Correia et al. (2010) demonstrated that VB on the surface of HTLV-1 infected cells or eluted from cells are highly infectious (Pais-Correia et al., 2010). Using a panel of lectins and MAbs these authors have also determined glycoproteins that are concentrated at viral assemblies, particularly, the extracellular matrix proteins collagen and agrin, the linker proteins tetherin and galectin-3. In our previous study, we have shown the importance of O-glycan bearing molecules, CD43 and CD45, for cell adhesiveness and transmission of HTLV-1 (Mazurov et al., 2012). As VB structures are enriched with highly glycosylated proteins, we believe that our published data support the model of virus transmission via VB. 
Although the role of VB in HTLV-1 infection has been demonstrated, molecular constituents of VB remain poorly investigated. In this study we used MAb screening technique to determine protein composition of VB. First, using electrophoretic separation of proteins we showed that VB eluted from the surfaces of HTLV-1 chronically infected cell lines, C91/PL or MT2, contain proteins that are not detected in viral particles isolated from the same cells. Second, by immunizing mice with isolated VB or with intact HTLV-1 infected cells we generated new MAbs 2C11, BF4, BF11, and $\mathrm{BF} 12$ that are raised against viral assemblies on the surfaces of HTLV-1 transformed cells. Two of them, BF11 and BF12, were identified as MAbs that recognize $\alpha$-chain of interleukin 2 receptor (IL-2RA or CD25). Third, we screened more than a hundred of CD MAbs with known specificities to the human lymphocyte antigens and found that CD4, CD70, CD80, CD150 antigens, as well as tetraspanin proteins CD9, CD53, CD63, and CD82 are enriched in VB.

Viral protein Tax plays a central role in HTLV-1 infection by coordinating the complex mechanisms of viral transmission (Nejmeddine and Bangham, 2010). We examined the levels of CD antigen expression on Jurkat T cells transduced with Tax. Consisting with the previous reports, we found the inducible patterns of expression for CD25 (Ballard et al., 1988; Ruben et al., 1988), CD54 (Mori et al., 1994), and CD70 (Baba et al., 2008) antigens. In addition, we found that Tax induced expression of CD80, a novel observation. In summary, using MAb screening we have determined cell surface molecules that are concentrated at VB and/or upregulated in T cells infected with HTLV-1. The mechanisms of their mobilization at VB and their role in HTLV-1 infection need to be delineated in further experiments.

\section{Materials and Methods}

Cell cultures and reagents. The human cell lines Jurkat E6-1, HEK 293T, and mouse myeloma cell line Sp2/0 were purchased from ATCC. The HTLV-1 transformed cell line MT2 was obtained through the NIH AIDS Research and Reference Reagent Program, and C91/PL cell line was kindly provided by Dr. Andres Alcover (Pasteur Institute). The suspension cells except Sp2/0 were maintained in RPMI culture medium supplemented with $10 \%$ fetal bovine serum, $2 \mathrm{mmol} / \mathrm{l} \mathrm{L}$-glutamine, and $40 \mu \mathrm{g} / \mathrm{ml}$ of gentamicin. HEK 293T and Sp2/0 cells were grown in DMEM culture medium containing $10 \%$ fetal bovine serum, $50 \mu \mathrm{mol} / \mathrm{l} \beta$-mercaptoethanol, $2 \mathrm{mmol} / \mathrm{l} \mathrm{L}$-glutamine, and $40 \mu \mathrm{g} / \mathrm{ml}$ of gentamicin. The primary anti-human CD MAbs (Sorbent, Russia) previously generated in the lab are listed at www.sobren.ru/prise.html. The rabbit polyclonal anti-HTLV-1 p19 (MA) Ab SP-61 raised against a peptide with the sequence of residues 102 to 115 of HTLV-1 Gag was a gift from Gisela Heidecker (NCI-Frederick, USA). The secondary antibodies were goat anti-rabbit conjugated to Alexa Fluor 350 and 546
(Molecular Probes), goat anti-mouse conjugated to Alexa Fluor 488 (Molecular Probes), goat anti-mouse conjugated to phycoerythrin (PE) (SantaCruz), and horseradish peroxidase-conjugated antimouse IgG (Cell Signaling, USA). The concanavalin A (ConA)-tetramethylrhodamine conjugate and rhodamine $6 \mathrm{G}$ were purchased from Molecular Probes and Syntol (Russia), respectively.

Isolation of viral particles and biofilms. HTLV-1 transformed cell lines MT2 and C91/PL were grown in $50 \mathrm{ml}$ of complete culture medium for 2-3 days. To make viral particle preparations approximately $2 \times 10^{7}$ cells were pelleted at $300 \mathrm{x}$ g for $10 \mathrm{~min}$, the supernatant was centrifuged at 3,000 $\mathrm{x}$ g for $30 \mathrm{~min}$ to remove cell debris, then filtered through a $0.22 \mu \mathrm{m}$ pore size filter, and finally centrifuged at $100,000 \mathrm{xg}$ for $1 \mathrm{hr}$ to pellet virions. In order to elute VB from the surface of HTLV-1 transformed cells, cells were first gently washed once with warm RPMI medium without serum, resuspended in $15 \mathrm{ml}$ RPMI supplemented with $10 \mathrm{mmol} / \mathrm{l} \mathrm{HEPES}$ buffer and $50 \mu \mathrm{g} / \mathrm{ml}$ of heparin and incubated at $37^{\circ} \mathrm{C}$ for $1 \mathrm{hr}$ as described earlier (Pais-Correia et al., 2010). Then cells were vigorously pipetted and pelleted at $300 \mathrm{x}$ g for $10 \mathrm{~min}$. The VB-containing supernatant was centrifuged at 3,000 $\mathrm{x}$ for $30 \mathrm{~min}$ to remove cell debris, and finally pelleted at 25,000 $\mathrm{x}$ g for $1 \mathrm{hr}$.

Generation and screening of MAbs. The pelleted VB prepared from $2 \times 10^{7}$ cells (per mouse) was resuspended in $0.25 \mathrm{ml}$ of PBS, emulgated with $0.25 \mathrm{ml}$ of complete Freund's adjuvant, and used for intra peritoneal immunization of 8 -week-old female BALB/c mice three times every 2 weeks. Alternatively, $5 \times 10^{6} \mathrm{C} 91 / \mathrm{PL}$ or MT2 cells (per mouse) gently washed once with warm RPMI medium were suspended in $0.5 \mathrm{ml}$ of PBS, and used for mice immunization. At the day 35 , test bleeds were performed, and sera from individual mice were tested by immunofluorescence and flow cytometry analysis. Mice whose sera had the highest levels of binding with MT2 or C91/PL cells were selected for the final booster immunization on the day 42. Four days after the final immunization, mice were euthanized; spleen lymphocytes were hybridized with the Sp2/0 myeloma cell line and cloned. The hybridoma cells were grown on a monolayer of feeder cells (mouse peritoneal macrophages) for about 2 weeks. The initial screening of hybridoma supernatants was performed using immunofluorescence and flow cytometry to select hybridomas positive for MT2 or C91/PL cells. The selected hybridoma supernatants were evaluated using fluorescence microscopy to select ones that predominantly stain VB on the surface of HTLV-1 transformed cells. All animal experiments described in this study conformed to the guidelines outlined in the Guide for the Care and Use of Laboratory Animals (NRC 2011) and were approved by the Bioethics Committee of the Institute of Immunology, Moscow, Russia.

Gel staining, immunoprecipitation, Western blotting, and massspectrometry analysis. The pellets of viral particles or VB (see preparations above) were solubilized in $50 \mu \mathrm{l}$ of $1 \mathrm{x}$ SDS sample buffer (Invitrogen), heated at $80^{\circ} \mathrm{C}$ for 5 min, separated using $12 \%$ polyacrylamide gel electrophoresis (PAGE), stained with Coomassie Blue, and imaged using Molecular Imager ChemiDoc XRS instrument (Bio-Rad). For immunoprecipitation (IP), MT2 or C91/PL cells 
were lysed in $1 \mathrm{ml}$ of ice-cold lysis buffer containing $1 \%$ Triton X-100 (Sigma), $150 \mathrm{mmol} / \mathrm{l} \mathrm{NaCl}, 10 \mathrm{mmol} / \mathrm{l}$ Tris ( $\mathrm{pH} \mathrm{8.0)}$ ), and a protease inhibitor cocktail (Complete Mini; Roche Applied Science). After 1 hour incubation at $4^{\circ} \mathrm{C}$, insoluble material was removed by centrifugation at $14,000 \mathrm{xg}$ for $10 \mathrm{~min}$. Cell lysates were first precleared with Protein A/G-agarose beads (Santa-Cruz) at $4^{\circ} \mathrm{C}$ for $1-2 \mathrm{hr}$, and then precipitated in the presence of Protein A/G-agarose beads loaded with specific MAb. Precipitation was carried out overnight at $4^{\circ} \mathrm{C}$ with constant rotation. After rinsing four times with ice-cold lysis buffer, immune complexes were eluted by heating at $80^{\circ} \mathrm{C}$ in SDS sample buffer, and resolved by $12 \%$ PAGE. Alternatively, SDS sample buffer was added to the cell lysate, the proteins were heat-denatured, and loaded on the gel with or without a reducing agent. Proteins from the gel were transferred onto Immobilon PVDF membrane (GE Healthcare). Western blots (WB) were probed with primary and then with horseradish peroxidase-conjugated antibodies. Immunoreactive bands were detected with Immobilon Western reagent (Millipore) using ChemiDoc XRS molecular imager (Bio-Rad). Fluorescent immunoprecipitation analysis (FIPA) combined with subsequent mass-spectrometry (MS) analysis was described earlier (Filatov et al., 2007). Briefly, $2 \times 10^{7} \mathrm{MT} 2$ cells were pelleted, washed twice with PBS, and labeled with $0.3 \mathrm{mg}$ of rhodamine $6 \mathrm{G}$ in $1 \mathrm{ml}$ of buffer containing $150 \mathrm{mmol} / \mathrm{l} \mathrm{NaCl}$ and $10 \mathrm{mmol} / \mathrm{l} \mathrm{NaHCO}{ }_{3}(\mathrm{pH}$ 8.0) for $20 \mathrm{~min}$. The reaction was stopped by adding $10 \mathrm{mmol} / \mathrm{l}$ glycine in PBS. Cells were washed with PBS, lysed, immunoprecipitated and resolved by $12 \%$ PAGE, as described above. The gel was analyzed with a laser-based fluorescence scanner Molecular Imager FX (Bio-Rad, USA). For MS identification of proteins, the major protein bands were excised, digested in-gel with trypsin, and analyzed by reversephase nano-LC-MS/MS with an Agilent 1100 nanoflow LC system coupled to an Agilent 1100 SL Series MSDTrap (Agilent Technologies, Alpharetta, GA, USA). Protein identification was performed with MASCOT software, and all tandem mass spectra were searched against the NCBI non-redundant database.

Lentiviral transduction. The Jurkat E6-1 cells were transduced with lentiviral vector encoding Tax essentially as described (Mazurov et al., 2010). Briefly, 293T cells were cotransfected with three plasmids: transfer vector pUCHR-Tax-IRES-GFP (Mazurov et al., 2010) encoding wild type (Wt) HTLV-1 Tax, IRES, and green fluorescent protein (GFP) gene under CMV promoter; HIV-1 packaging plasmid pCMV $\triangle 8.2 \mathrm{R}$ (Addgene); and pCMV VSVG vector (Addgene) encoding env from vesicular stomatitis virus (VSV) at a 6:4:0.5 ratio, respectively. Cells were transfected overnight with Lipofectamine 2,000 reagent (Invitrogen) according to the manufacturer's instructions. Next day the culture medium was replaced. The supernatant containing virus-like particles (VLPs) was harvested $48 \mathrm{hrs}$ post-transfection and filtered through a 0.45 $\mu \mathrm{m}$ pore size filter (Corning). Jurkat cells $\left(10^{7}\right)$ were infected with lentiviral particles at a multiplicity of infection (MOI) of $\sim 0.5$. Three days later the transduced cells were harvested, stained with Abs, and analyzed by flow cytometry.

Cas9-mediated gene knockout. In order to knockout IL-2RA in MT2 cells we used clustered regularly interspaced short palindromic repeats (CRISPR)-Cas9 and guide-RNA (gRNA) mediated gene knockout (Mali et al., 2013) in combination with double nicking (DN) (Ran et al., 2013a; Ran et al., 2013b). The gRNA target sequences 5'-CAGCCCCAGCTCATATGCAC-3' and 5'-GGTCCACCTTGTCTTCCCGT-3' corresponding to exon 4 of IL-2RA were selected using the web-based resource http://tools. genome-engineering.org. The gRNA expressing vector $\mathrm{pKS}$ gRNA $\mathrm{BB}$ was constructed by subcloning U6-gRNA expression cassette from pCR-BlunII-TOPO gRNA vector (Addgene \#41824) into pBluescript II KS (+) vector (Stratagen) at BstXI and PstI sites of restriction followed by substitution of Afl II cloning site with a double Bbs I site. The annealed oligos containing target sequences were cloned into pKS gRNA BB plasmid after digestion with Bbs I restrictase. The D10A nickase mutant of Cas9 was generated in pcDNA 3.3 TOPO hCas9 plasmid (Addgene \#41815) by inserting mutation-bearing oligos between $\mathrm{XbaI}$ and $E c o$ RV sites. MT2 cells $\left(10^{5}\right)$ were cotransfected with a pair of gRNA vectors ( $3 \mu \mathrm{g}$ of each plasmid) and $3 \mu \mathrm{g}$ of Cas9 D10A expression vector by using Neon electroporation system (Invitrogen) (1 pulse of $40 \mathrm{~ms}$ at $1250 \mathrm{~V}$ ). Cells were grown for 2-3 weeks, and analyzed for CD25 expression using flow cytometry. CD25-negative cells were sorted out twice on FACSAria II Becton Dickinson Instrument to enrich the population of knockout cells above $99 \%$.

Immunofluorescence microscopy and flow cytometry. MT2 or C91/PL cells were washed with PBS and adhered to poly-L-lysine (Sigma)-coated coverslips for $20 \mathrm{~min}$. The cells were then fixed with $4 \%$ paraformaldehyde (Sigma) in PBS for $10 \mathrm{~min}$, washed with PBS and immediately stained with mouse MAb for 40 min. In some experiments plasma membrane was stained with rhodamine-labeled ConA that was added to primary MAb solution at a final concentration of $50 \mu \mathrm{g} / \mathrm{ml}$. Cells were washed with PBS twice, and stained with secondary goat anti-mouse Alexa Fluor $488 \mathrm{Ab}$ for $40 \mathrm{~min}$. After washing, cells were permeabilized with PBS containing $0.1 \%$ saponin (Sigma) and $1 \%$ fetal bovine serum. The HTLV-1 matrix protein was stained with a polyclonal rabbit anti-p19 Ab that was added directly to the permeabilization solution for $1 \mathrm{hr}$ and then washed with permeabilization solution twice. Secondary goat anti-rabbit Alexa Fluor 350 or $546 \mathrm{Ab}$ was added to the permeabilization solution in the presence of $1 \%$ normal goat serum for $40 \mathrm{~min}$. After washing, the coverslips were transferred onto slides and maintained in Dako Cytomation Fluorescent Mounting Medium. Images were taken with Nikon Eclipse TE2000 confocal microscope. Image data were processed with Fiji image analysis software. Colocalization of cell surface antigen with VB (p19 staining) was estimated as the Pearson coefficient of correlation (1.0 is full colocalization, 0 is no colocalization) for cross-sections of individual cells. Live cells in suspension were immunostained with primary MAb and secondary phycoerythrin-labeled Ab according to a standard protocol. Samples were analyzed with a FACScan flow cytometry instrument (Becton Dickinson) and CELL QUEST software. The FACS results were presented as histograms and dot plots using WinMDI 2.8 software. 


\section{Results}

Viral biofilms isolation and characterization

To isolate VB we used an earlier described method that utilizes heparine for elution of VB from the surfaces of HTLV-1 transformed cells (Pais-Correia et al., 2010). The viral particles produced by HTLV-1 infected cells were purified according to a standard protocol. The step-by-step procedures for the virus and VB isolation are schematically presented in Fig. 1a, and described in details in Materials and Methods. Samples of viral particles and VB containing equal amounts of total protein were resolved by $12 \%$ PAGE and stained with Coomassie dye (Fig. 1b, lanes 1 and 3, respec-

(a)

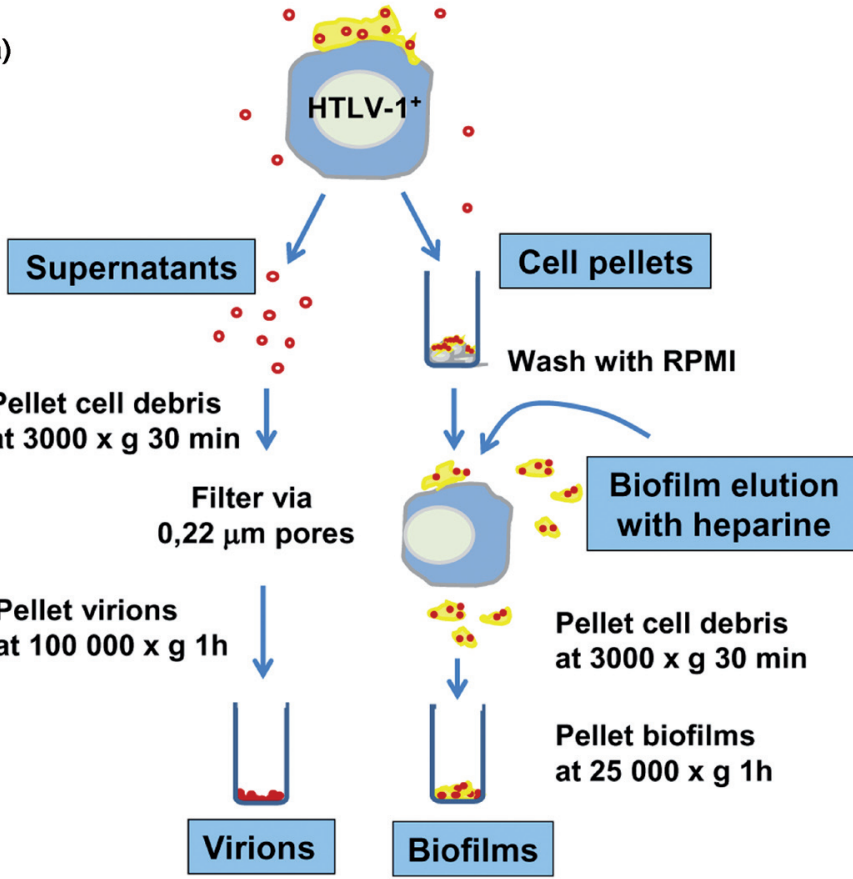

(b)

(c)

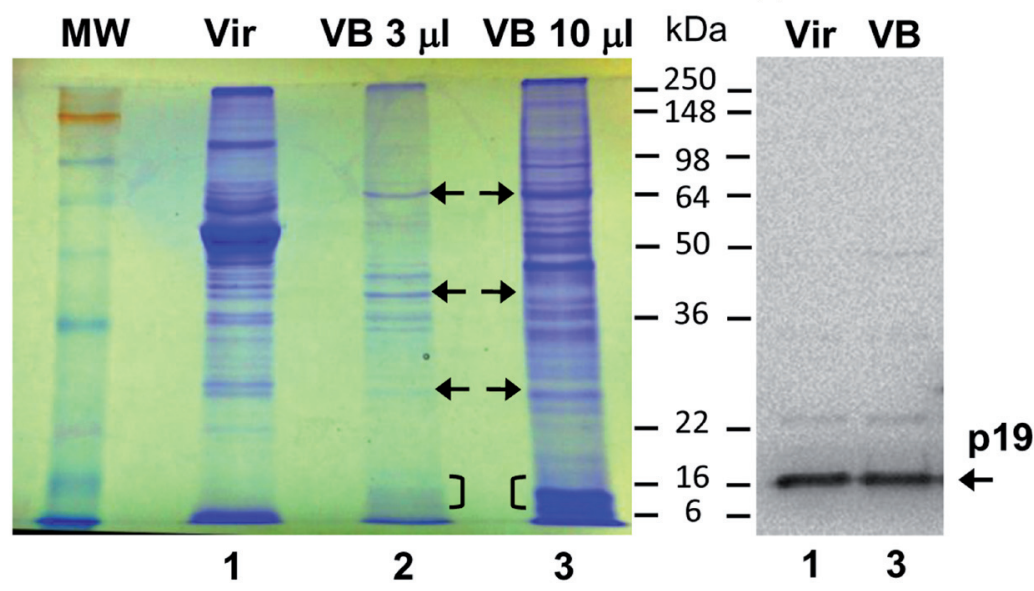

Fig. 1

Isolation and characterization of HTLV-1 VB

(a) Schematic presentation of step-by-step isolation procedure for viral biofilms and cell-free virions. (b) Comparative analysis of Coomassie-stained proteins extracted from VB and cell-free virions (Vir), and resolved by 12\% PAGE under non-reducing conditions. (c) Western blot analysis of VB and virions stained for the HTLV-1 p19 (matrix) protein (non-reducing conditions). Representative images of three experiments are shown in panels (b) and (c). 
tively). In addition, threefold lower amount of VB sample was loaded on the gel (Fig. 1b, lane 2). As indicated by arrows, VB sample contains protein bands with electrophoretic mobility different from that of proteins in cell-free viral particles. Both samples, cell-free viruses and VB, contained approximately the same amounts of viral proteins, as demonstrated on the Western blot stained for HTLV-1 core protein p19 Gag (Fig. 1c, corresponds to lanes 1 and 3 in Fig. 1b). Thus, VB isolated from HTLV-1 positive cells have a different protein composition than cell-free virions.

\section{Generation and identification of MAbs raised against VB}

In order to decipher the protein composition of $\mathrm{VB}$, we decided to generate MAbs against VB. Mice were immunized either with isolated VB or with intact HTLV-1 infected cells bearing VB on their surfaces. Immunization with VB in Freund's adjuvant or with cells was carried out as outlined in Materials and Methods. The generated hybridoma clones were exposed to two rounds of screening analysis. In the first round, using immunofluorescence and flow cytometry, we selected hybridoma supernatants that bind to the surface of C91/PL or MT2 cells; more than 50 positive hybridomas were identified. During the second round of hybridoma screening we identified MAbs that predominantly stained VB on the surfaces of HTLV-1 infected cells. For that purpose the degree of colocalization between intracellularly stained Gag (marker of VB) and surface antigen stained with MAb was estimated by fluorescence microscopy. To show that VB stained for Gag are on the plasma membrane and are enriched with glycoproteins, cell surfaces were costained with ConA in some samples. We identified two MAbs, BF4 and 2C11, that predominantly stained the VB. One of the MAbs (BF5) evenly stained the surface of C91/ PL and MT2 cells and, therefore, was chosen as a negative control. For mice immunized with whole cells, two MAbs, BF11 and BF12, were obtained after the screening of about 150 hybridoma clones, which were positive in binding to HTLV-1 cells. The representative confocal images of C91/ PL and MT2 cells stained with these MAbs, along with the Pearson coefficient of correlation $\mathrm{R}$ as a measurement of colocalization of MAb with VB, are shown in Fig. 2. The $\mathrm{R}$ values for colocalization of Gag and anti-VB MAbs BF4, $\mathrm{BF} 11$, and BF12 ranged from 0.76 to 0.85 while the $\mathrm{R}$ value for control MAb BF5 was below 0.2.

Next, we characterized the newly generated MAbs by the pattern of expression on different types of cells. As demonstrated in Table 1, the epitopes of BF5, BF11, and BF12 MAbs were exclusively expressed on the surfaces of HTLV-1 positive cells, whereas the BF4 MAb also stained many populations of lymphoid and myeloid cells except NK-lymphocytes. As MAb BF4 did not work in IP or WB, its specificity was not identified. The hybridoma 2C11 was unstable and stopped MAb production over time.

MAbs BF11 and BF12 performed well in both IP and WB procedures. As shown by IP of rhodamine-6G labeled (a)

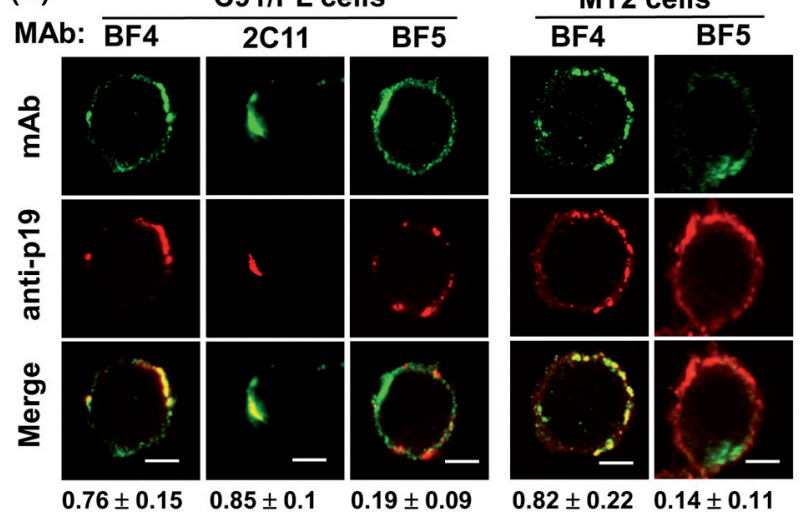

(b)

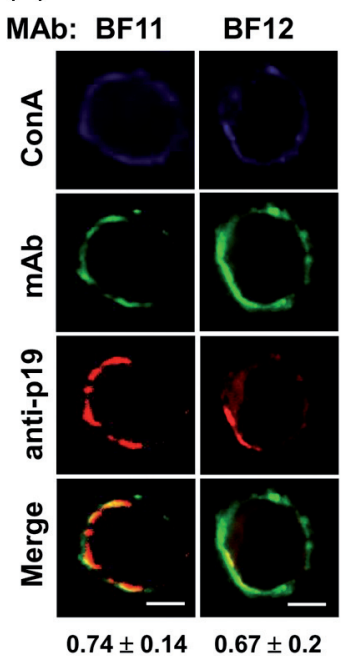

Fig. 2

Immunostaining of $\mathrm{VB}$ with newly generated MAbs

MAbs were generated after immunization with VB (a) or with whole C91/PL cells (b). Surface of MT2 or C91/PL cells was stained with indicated MAb (green) and ConA (blue in B), permeabilized, and stained with anti-p19 Ab (red). The Pearson coefficients of correlation R, as a value of colocalization between MAb and VB (p19) are shown at the bottom of each picture. R values represent averages of 3 to 5 measurements with standard deviations. Representative confocal images of optical slices through the middle of the cells are shown. Scale bar, $5 \mu \mathrm{m}$. 
Table 1. Binding of the newly generated MAbs to the surface of different cell types

\begin{tabular}{lcccc}
\hline Cells / MAbs & BF5 & BF4 & BF11 & BF12 \\
\hline C91PL (HTLV-1+) & ++++ & ++++ & ++++ & ++++ \\
MT2 (HTLV-1+) & ++++ & ++++ & ++++ & ++++ \\
Neutrophils & - & + & - & - \\
Monocytes & - & + & - & - \\
T cells & - & +++ & - & - \\
B cells & - & +++ & - & - \\
NK cells & - & - & - & - \\
\hline
\end{tabular}

proteins (Fig. 3a), both of these MAbs precipitated an antigen of a similar molecular weight of 50-55 kDa. These MAbs also stained an antigen of $50-55 \mathrm{kDa}$ on a WB of whole cell lysates or after IP under non-reducing conditions (Fig. 3b). The bands indicated in Fig. 3a were excised from the gel, trypsinized, and analyzed by MS. MS analysis identified in both samples the $a$-chain of the interleukin 2 receptor (IL2RA or CD25). To gain an additional proof that BF11 and $\mathrm{BF} 12$ recognize $\mathrm{CD} 25$, we generated an MT2 cell line with CD25 knockout (KO) using the recently described method of Cas9-mediated double nicking (Ran et al., 2013a,b). These CD25-knockout $(\mathrm{KO})$ and Wt MT2 cells were stained with
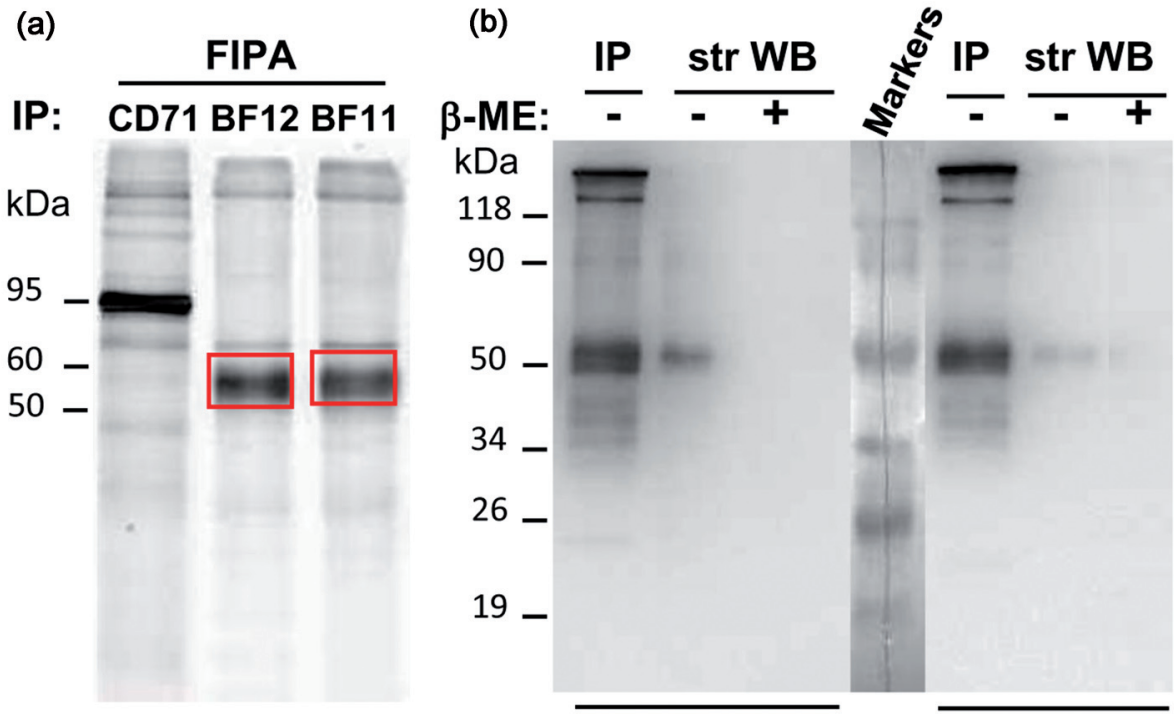

WB:

BF12

BF11

(c)
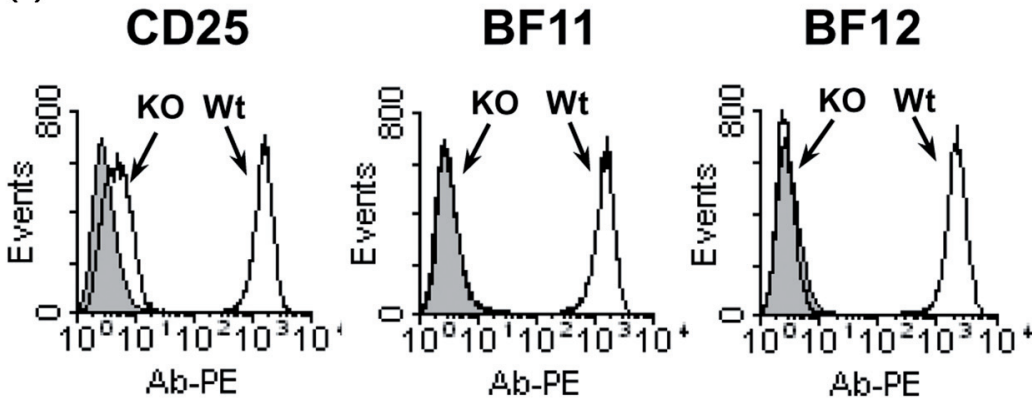

Fig. 3

Identification of MAbs raised against VB

(a) Fluorescent immunoprecipitation analysis of proteins pulled down with anti-CD71 (control), BF11, and BF12 MAbs. Proteins excised from the gel (red boxes) were analyzed by mass-spectrometry. (b) Western blot analysis of proteins after IP with BF12 or BF11 MAb or directly from whole cell lysates (WB). Samples were resolved by $12 \%$ PAGE under reducing (with $\beta$-ME, $\beta$-mercaptoethanol) or non-reducing conditions as indicated, then blotted and probed with BF12 or BF11 MAb. (c) FACS analysis of anti-CD25, BF11 and BF12 MAb binding to the surface of Wt or CD25 knockout (KO) MT2 cells (pointed by arrows). The controls without primary $\mathrm{Ab}$ are presented in filled histograms. Representative images of three experiments are shown. 
anti-CD25, BF11, or BF12 MAbs followed by probing with anti-mouse PE labeled Ab, and analyzed by FACS. As demonstrated on histograms (Fig. 3c), all three MAbs showed

(a)

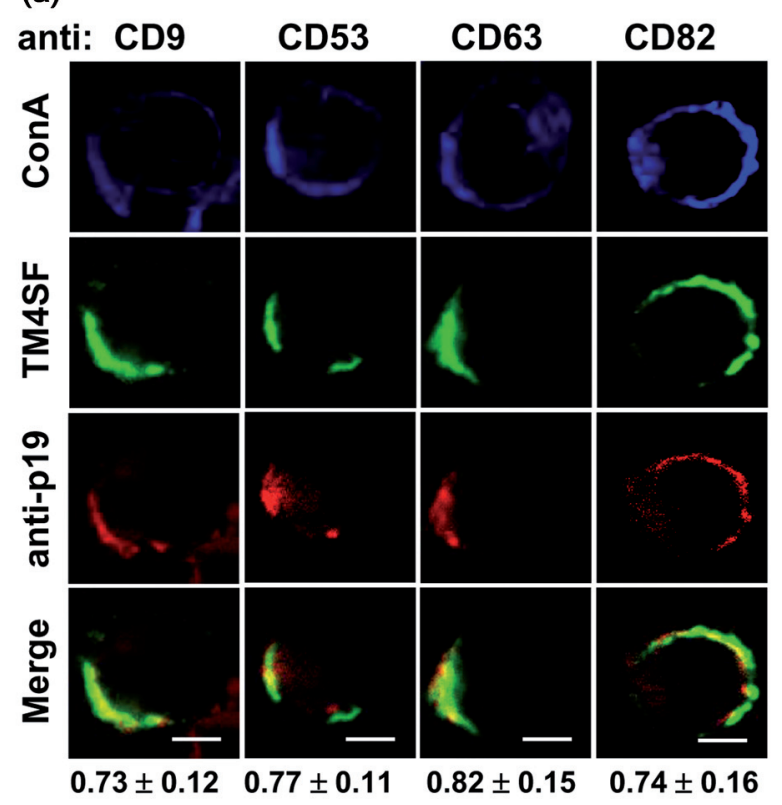

(b)

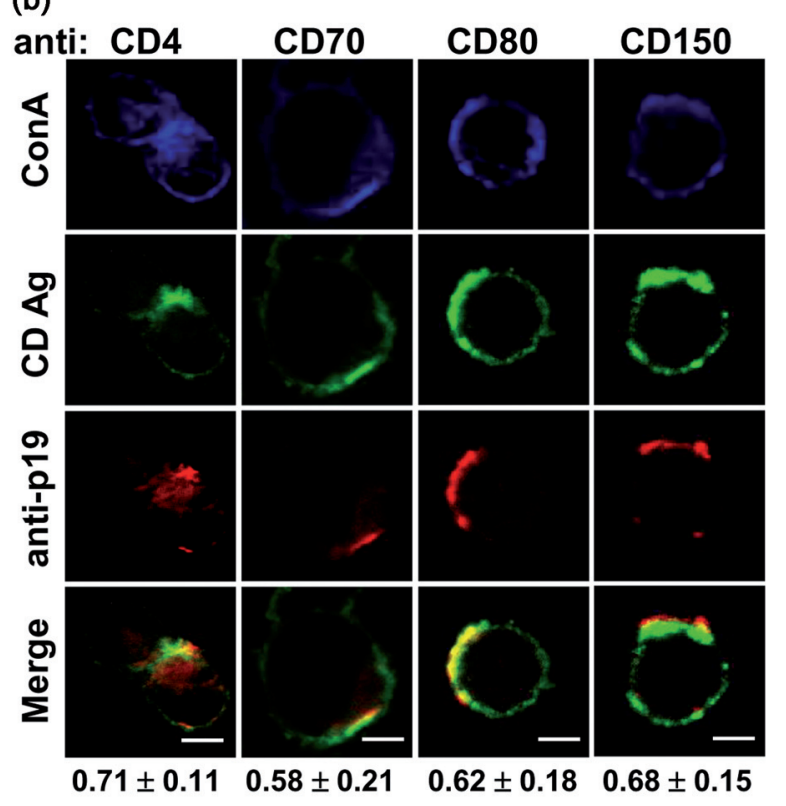

Fig. 4

VB-associated antigens selected by the screening of CD MAbs with known specificities

(a) A group of proteins from the tetraspanin family (TM4SF). (b) Other proteins (CD Ag) from the different families. MT2 cells were stained with ConA and antibodies, as described in Fig. 2b. R values of colocalization between MAb and VB are shown at the bottom of each panel; values represent averages of 4 to 5 independent experiments with standard deviations. Scale bar, $5 \mu \mathrm{m}$. similar levels of staining of Wt MT2 cells, and no binding with KO MT2 cells. These data confirm the specificity of BF11 and BF12 to CD25 antigen.

In conclusion, we generated four MAbs, which specifically stained HTLV-1 VB, and identified CD25 antigen as associated with VB.

Using MAbs with known specificities to identify CD antigens associated with $\mathrm{VB}$

In order to find more antigens associated with VB, we screened a panel of MAbs against human CD antigens with known specificities using colocalization test described above. The panel of MAbs that were previously generated and identified in our lab is listed at www.sobren.ru/prise.html. In total, more than hundred MAbs were tested. The antigens that we selected by this screening were divided in two groups. The first group was comprised of tetraspanin proteins (TM4SF) CD9, CD53, CD63, and CD82, and showed high level of colocalization with VB (Fig. 4a). Previously we demonstrated that CD82 and other TM4SF proteins interact directly with HTLV-1 Gag (Mazurov et al., 2006, 2007); therefore, the proteins from this family are likely to be recruited into the membrane of virions, but do not constitute the matrix of VB that surrounds viral particles. The second group included the antigens of different origins (CD4, CD70, CD80, and CD150) with lower levels of colocalization to VB (Fig. 4b). The recruitment of these antigens into VB has not been reported earlier, and their exact localization in VB structures is unknown. Thus, by screening a panel of MAbs with known specificities we found four new antigens, CD4, CD70, CD80, and CD150, that associate with VB.

\section{Identifying antigens that are upregulated by Tax expression}

The viral protein Tax plays a pleotropic role in the biology of HTLV-1. Particularly, the initiation and elongation of viral transcription and leukemic transformation of infected T lymphocytes are widely recognized functions of Tax (de la Fuente and Kashanchi, 2004). Tax enhances cell-to-cell transmission of HTLV-1 by the mechanisms of cytoskeleton reorganization, induction of ICAM-1 expression and cell adhesion, and likely by other not yet recognized mechanisms (Mazurov et al., 2010). It is possible that Tax is also involved in VB-mediated cell-to-cell transmission of HTLV-1, therefore, we aimed to find the expression of which cell surface proteins depends on Tax. Lentiviral vector carrying a cassette that expresses Tax-IRES-GFP was used to transduce CD4+ Jurkat cells at MOI of $\sim 0.5$. At this MOI roughly $50 \%$ of the cells remained Tax-negative and served as an internal control. Cells were immunostained with various MAbs and analyzed by flow cytometry. Markers specific for B cells and NK cells lineage were excluded from this analysis. The examples of 
Tax-inducible (CD25, CD54 and CD70) and non-inducible (CD47) antigens are presented in Fig. 5a. The ratio of mean fluorescence intensity (MFI) in PE channel of Tax-positive cells (region R3) to the MFI of Tax-negative cells (region R2) was calculated and plotted in Fig. 5b. As shown, the majority of tested CD antigens were not upregulated in Tax expressing cells. However, the expression levels of CD25, CD54 (ICAM-1), and CD70 on the surface of these cells were about 8,13 , and 6 -fold higher, respectively, than on the surface of non-transduced Jurkat cells. Expression of CD80 and CD95 was only mildly boosted by Tax expression (roughly two-fold), while expression of CD162 was downregulated in Tax-positive cells.

In summary, using MAbs screening we determined cell surface antigens, i.e. CD25, CD70, and CD80, that are both associated with VB and upregulated by Tax. ICAM-1 was significantly overexpressed in cells transduced with Tax, but evenly distributed on surface of HTLV-1 positive cells and did not selectively associate with VB. Conversely, CD4 and
CD150 were concentrated at VB, but expressed independently of Tax.

\section{Discussion}

Primary infection and consequent spreading of HTLV-1 in vivo are mediated by complex mechanisms of cell-to-cell viral transmission (Nejmeddine and Bangham, 2010; Pique and Jones, 2012) The pivotal role in this process belongs to HTLV-1 Tax protein, which coordinates cytoskeleton polarization, induces expression of adhesion molecule ICAM-1, promotes VS formation, and can potentially regulate VB-mediated viral transfer (Nejmeddine and Bangham, 2010). Interacting with transcription factors, signaling and transport molecules, cytoskeleton, PDZ proteins and other intracellular messengers, Tax induces global changes in the cellular transcriptome (reviewed in (Boxus et al., 2008)) as was demonstrated at the level of mRNA (Baba et al., 2008;

(a)

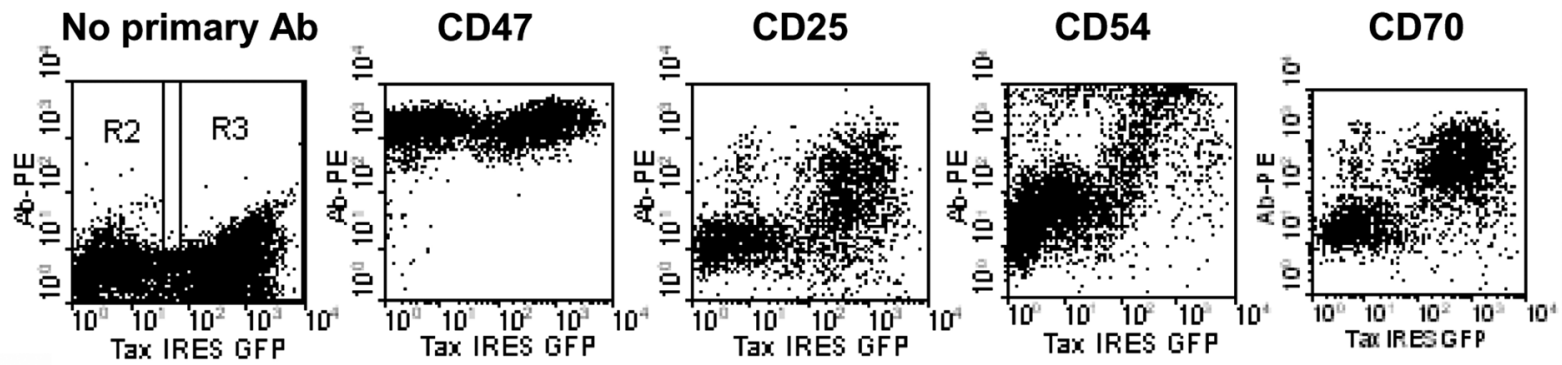

(b)

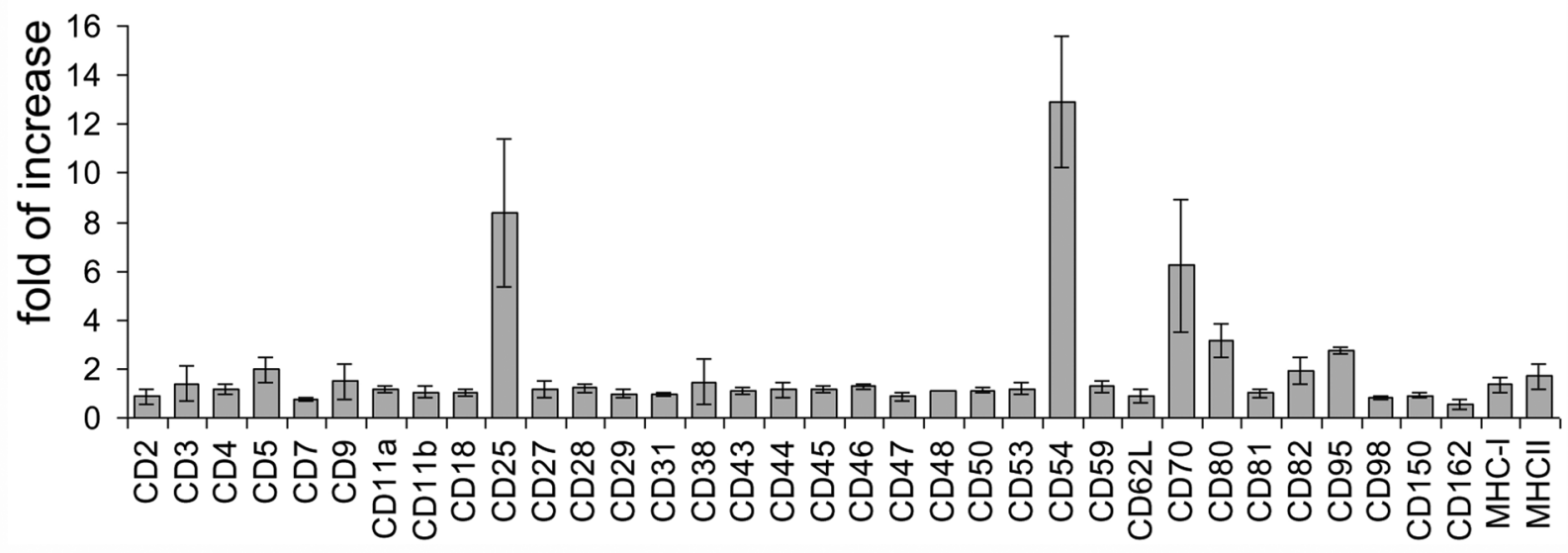

Fig. 5

Expression of CD antigens on the surface of Jurkat cells transduced with HTLV-1 Tax

(a) Typical dot plots demonstrating Tax-independent (CD47) or Tax-inducible (CD25, CD54, CD70) patterns of expression. (b) The levels of CD antigen expression in Tax-positive cells relative to Tax-negative cells were calculated as the mean fluorescence intensity for PE in region 3 (R3) divided by the mean fluorescence intensity for PE in region 2 (R2); gating for regions R2 and R3 is shown in the left panel of Fig. 5a. The averages of three independent experiments with standard deviations are shown. 
Ng et al., 2001) and cell surface protein expression (reviewed in (Kress et al., 2011)).

In our study, using MAbs screening technique we have determined cell surface proteins that are associated with the HTLV-1 VB structures and that are upregulated by Tax expression. We found that CD25, CD70, and CD80 are both localized at VB and upregulated by Tax. The Tax-inducible expression of these antigens has been reported earlier (Baba et al., 2008; Ballard et al., 1988; Dezzutti et al., 1995). However, the role of such upregulation in HTLV-1 pathogenesis remains unknown. Interestingly, even though HTLV-1 transformed cell lines express high level of IL-2 receptor a (CD25), most of them do not produce IL-2 (Arya et al., 1984). This observation rules out the hypothesis of autocrine IL-2 dependent proliferation of HTLV-1 transformed cells. CD70 and CD80 (B7-1) are ligands for costimulatory receptors CD27 (TNFRSF7) and CD28, respectively. CD80 also binds to CTLA4, an inhibitory receptor of T cells. Both ligands may regulate the cell's activation status by mechanisms that are not clear, because, for instance, CD27 is not expressed on HTLV-1 positive cells. In contrast to autocrine mechanisms, the expression and localization of these antigens at VB can be essential for the viral transmission upon contact of an HTLV-1 infected cell with a healthy target T cell. The other antigens selected in our screening assays, CD4 and CD150, are localized at VB, but expressed independently of Tax. CD150 is a signaling lymphocyte activation molecule (SLAM) with self-activation property and, therefore, can be involved in homotypic cell activation. Remarkably, ICAM-1 (CD54), a key adhesion molecule that is recruited into immunological and virological synapses (Vasiliver-Shamis et al., 2010), was strongly upregulated by Tax, but remained evenly distributed on the surfaces of HTLV-1 transformed cells. This may suggest that infected cell can form an adhesion contact with target cell outside of VB region.

At the current stage, we do not know whether the VBassociated antigens are important for $\mathrm{VB}$ formation and HTLV-1 infection, and whether they are accumulated in the matrix of $\mathrm{VB}$ or incorporated into the membrane of virions. It is assumed that assemblies of viral particles on the surfaces of HTLV-1 infected cells are protected by the matrix components of VB. Therefore, for efficient viral transmission, there should be some mechanisms of reception and endocytosis of VB by the target cells. In other words, HTLV-1 env cannot interact with its receptors unless the VB components are disintegrated in cellular endosomal compartments. Such hypothetical mechanism suggests the existence of VB-specific cell surface receptors, which are different from the receptors described for HTLV-1 such as glucose transporter 1(GLUT-1), beuropilin 1 (NRP-1) and heparan sulphate proteoglycans (HSPG) (Ghez et al., 2010). Thus, the search for ligands (molecular components of VB) and their counter-receptors on target cells may help us to better understand the mechanisms of HTLV-1 transmission and entry.

In conclusion, using MAb screening we have determined new antigens (CD4, CD25, CD70, CD80, and CD150) that are concentrated in HTLV-1 VB. The role of these antigens in VB integrity and HTLV-1 infection is not clear at this time and must be examined in further studies.

Acknowledgements. This work has been supported by the grant of Russian Foundation for Basic Research (RFBR № 12-04-00415-a), partially by the NIH-RFBR grant № 13-04-91449, and by the grant of Russian Science Foundation (RSF № 15-15-00135). We thank Alexander Sapojnikov and Elena Kovalenko from the Institute of Bioorganic Chemistry (Moscow, Russia) for their help with confocal imaging, and Olga Nikolaitchik from NCI-Frederick (Frederick, $\mathrm{MD)}$ for critical reading of the manuscript.

\section{References}

Arya SK, Wong-Staal F, Gallo RC (1984): T-cell growth factor gene: lack of expression in human T-cell leukemia-lymphoma virus-infected cells. Science 223, 1086-1087. http:// dx.doi.org/10.1126/science.6320374

Baba M, Okamoto M, Hamasaki T, Horai S, Wang X, Ito Y, Suda Y, Arima N (2008): Highly enhanced expression of CD70 on human T-lymphotropic virus type 1-carrying T-cell lines and adult T-cell leukemia cells. J. Virol. 82, 3843-3852. http://dx.doi.org/10.1128/JVI.02013-07

Ballard DW, Bohnlein E, Lowenthal JW, Wano Y, Franza BR, Greene WC (1988): HTLV-I tax induces cellular proteins that activate the kappa B element in the IL-2 receptor alpha gene. Science 241, 1652-1655. http://dx.doi.org/10.1126/ $\underline{\text { science. } 2843985}$

Boxus M, Twizere JC, Legros S, Dewulf JF, Kettmann R, Willems L (2008): The HTLV-1 Tax interactome. Retrovirology 5, 76. http://dx.doi.org/10.1186/1742-4690-5-76

Chen BK (2012): T cell virological synapses and HIV-1 pathogenesis. Immunol. Res. 54, 133-139. http://dx.doi. org/10.1007/s12026-012-8320-8

de la Fuente C, Kashanchi F (2004): The expanding role of Tax in transcription. Retrovirology 1, 19. http://dx.doi. org/10.1186/1742-4690-1-19

Dezzutti CS, Rudolph DL, Lal RB (1995): Infection with human T-lymphotropic virus types I and II results in alterations of cellular receptors, including the up-modulation of T-cell counterreceptors CD40, CD54, and CD80 (B7-1). Clin. Diagn. Lab. Immunol. 2, 349-355.

Filatov AV, Krotov GI, Zgoda VG, Volkov Y (2007): Fluorescent immunoprecipitation analysis of cell surface proteins: a methodology compatible with mass-spectrometry. J. Immunol. Methods 319, 21-33. http://dx.doi.org/10.1016/j. jim.2006.09.014

Ghez D, Lepelletier Y, Jones KS, Pique C, Hermine O (2010): Current concepts regarding the HTLV-1 receptor complex. 
Retrovirology 7, 99. http://dx.doi.org/10.1186/17424690-7-99

Igakura T, Stinchcombe JC, Goon PK, Taylor GP, Weber JN, Griffiths GM, Tanaka Y, Osame M, Bangham CR (2003): Spread of HTLV-I between lymphocytes by virus-induced polarization of the cytoskeleton. Science 299, 1713-1716. http://dx.doi.org/10.1126/science.1080115

Jolly C, Kashefi K, Hollinshead M, Sattentau QJ (2004): HIV-1 cell to cell transfer across an Env-induced, actin-dependent synapse. J. Exp. Med. 199, 283-293. http://dx.doi. org/10.1084/jem.20030648

Kress AK, Grassmann R, Fleckenstein B (2011): Cell surface markers in HTLV-1 pathogenesis. Viruses 3, 1439-1459. http:// dx.doi.org/10.3390/v3081439

Mali P, Yang L, Esvelt KM, Aach J, Guell M, DiCarlo JE, Norville JE, Church GM (2013): RNA-guided human genome engineering via Cas9. Science 339, 823-826. http://dx.doi. org/10.1126/science. 1232033

Mazurov D, Heidecker G, Derse D (2006): HTLV-1 Gag protein associates with CD82 tetraspanin microdomains at the plasma membrane. Virology 346, 194-204. http://dx.doi. org/10.1016/i.virol.2005.10.033

Mazurov D, Heidecker G, Derse D (2007): The inner loop of tetraspanins $\mathrm{CD} 82$ and $\mathrm{CD} 81$ mediates interactions with human $\mathrm{T}$ cell lymphotrophic virus type $1 \mathrm{Gag}$ protein. J. Biol. Chem. 282, 3896-3903. http://dx.doi.org/10.1074/ jbc.M607322200

Mazurov D, Ilinskaya A, Heidecker G, Filatov A (2012): Role of $\mathrm{O}$-glycosylation and expression of CD43 and CD45 on the surfaces of effector T cells in human T cell leukemia virus type 1 cell-to-cell infection. J. Virol. 86, 2447-2458. http://dx.doi.org/10.1128/JVI.06993-11

Mazurov D, Ilinskaya A, Heidecker G, Lloyd P, Derse D (2010): Quantitative comparison of HTLV-1 and HIV-1 cell-tocell infection with new replication dependent vectors. PLoS Pathog 6, e1000788. http://dx.doi.org/10.1371/ journal.ppat.1000788
Mori N, Murakami S, Oda S, Eto S (1994): Human T-cell leukemia virus type I tax induces intracellular adhesion molecule-1 expression in T cells. Blood 84, 350-351.

Nejmeddine M, Bangham CR (2010): The HTLV-1 Virological Synapse. Viruses 2, 1427-1447. http://dx.doi.org/10.3390/ v2071427

Ng PW, Iha H, Iwanaga Y, Bittner M, Chen Y, Jiang Y, Gooden G, Trent JM, Meltzer P, Jeang KT, Zeichner SL (2001): Genome-wide expression changes induced by HTLV-1 Tax: evidence for MLK-3 mixed lineage kinase involvement in Tax-mediated NF-kappaB activation. Oncogene 20, 4484-4496. http://dx.doi.org/10.1038/sj.onc. 1204513

Pais-Correia AM, Sachse M, Guadagnini S, Robbiati V, Lasserre R, Gessain A, Gout O, Alcover A, Thoulouze MI (2010): Biofilm-like extracellular viral assemblies mediate HTLV-1 cell-to-cell transmission at virological synapses. Nat. Med. 16, 83-89. http://dx.doi.org/10.1038/nm.2065

Pique C, Jones KS (2012): Pathways of cell-cell transmission of HTLV-1. Front Microbiol 3, 378. http://dx.doi.org/10.3389/ $\underline{\text { fmicb.2012.00378 }}$

Ran FA, Hsu PD, Lin CY, Gootenberg JS, Konermann S, Trevino $\mathrm{AE}$, Scott DA, Inoue $\mathrm{A}$, Matoba S, Zhang $\mathrm{Y}$, Zhang $\mathrm{F}$ (2013a): Double nicking by RNA-guided CRISPR Cas9 for enhanced genome editing specificity. Cell 154, 1380-1399. http://dx.doi.org/10.1016/j.cell.2013.08.021

Ran FA, Hsu PD, Wright J, Agarwala V, Scott DA, Zhang F (2013b): Genome engineering using the CRISPR-Cas9 system. Nat Protoc 8, 2281-2308. http://dx.doi.org/10.1038/ nprot.2013.143

Ruben S, Poteat H, Tan TH, Kawakami K, Roeder R, Haseltine W, Rosen CA (1988): Cellular transcription factors and regulation of IL-2 receptor gene expression by HTLV-I tax gene product. Science 241, 89-92. http:// dx.doi.org/10.1126/science. 2838905

Vasiliver-Shamis G, Dustin ML, Hioe CE (2010): HIV-1 Virological Synapse is not Simply a Copycat of the Immunological Synapse. Viruses 2, 1239-1260. http://dx.doi.org/10.3390/v2051239 